\title{
Approximate Analytical Solutions of Nonlinear Korteweg-de Vries Equations Using Multistep Modified Reduced Differential Transform Method
}

\author{
Che Haziqah Che Hussin ${ }^{1,2, *}$, Ahmad Izani Md Ismail ${ }^{1}$, Adem Kilicman ${ }^{3}$, Amirah Azmi ${ }^{1}$ \\ ${ }^{1}$ School of Mathematical Sciences, University of Science, Malaysia \\ ${ }^{2}$ Preparatory Centre of Science and Technology, Universiti Malaysia Sabah, Malaysia \\ ${ }^{3}$ Department of Mathematics, Faculty of Science, Universiti Putra Malaysia, Malaysia
}

Received August 5, 2019; Revised December 22, 2019; Accepted February 20, 2020

Copyright $\bigcirc 2020$ by authors, all rights reserved. Authors agree that this article remains permanently open access under the terms of the Creative Commons Attribution License 4.0 International License

\begin{abstract}
This paper aims to propose and investigate the application of Multistep Modified Reduced Differential Transform Method (MMRDTM) for solving the nonlinear Korteweg-de Vries (KdV) equation. The proposed technique has the advantage of producing an analytical approximation in a fast converging sequence with a reduced number of calculated terms. MMRDTM is presented with some modification of the reduced differential transformation method (RDTM) which is the nonlinear term is replaced by related Adomian polynomials and then adopting a multistep approach. Consequently, the obtained approximation results do not only involve smaller number of calculated terms for the nonlinear $\mathrm{KdV}$ equation, but also converge rapidly in a broad time frame. We provided three examples to illustrates the advantages of the proposed method in obtaining the approximation solutions of the $\mathrm{KdV}$ equation. To depict the solution and show the validity and precision of the MMRDTM, graphical inputs are included.
\end{abstract}

Keywords Adomian Polynomials, Multistep Approach, Nonlinear Korteweg-de Vries Equation, Reduced Differential Transform Method

\section{Introduction}

Partial differential equations (PDEs) have broad applications in various branches of sciences and engineering such as fluid mechanics, thermodynamics, heat transfer as well as many other areas of physics [1]. For many nonlinear PDEs, it is rather challenging to manage the nonlinear terms of these equations. Despite the fact that most researches utilized numerical methods to obtain the approximate solution of these equations, being able to solve such equations analytically is significant due to the fact that manipulation are easier if the approximation is analytical in nature. Many PDEs and ordinary differential equations (ODEs) have been tackled by using approximate analytical methods such as Differential Transform Method (DTM) and Reduced Differential Transform Method (RDTM) [26]. There exists scope to seek improvements for the method.

Sahoo and Ray [7] obtained analytical solutions of time fractional modified Korteweg-de Vries (KdV) equation by using $\left(\mathrm{G}^{\prime} / \mathrm{G}\right)$-expansion method and improved $\left(\mathrm{G}^{\prime} / \mathrm{G}\right)$ expansion method. Besides that, Islam et. al [8] considered a recent extension of the $\left(G^{\prime} / G\right)$-expansion approach in determining the solitary wave solutions of the modified $\mathrm{KdV}$ equation. Apart from that, Ray [9] proposed a modification on the fractional RDTM and implemented it to find solutions of fractional $\mathrm{KdV}$ equations. In this approach, the adjustment included the substitution of the nonlinear term by relating Adomian polynomials [9]. Therefore, the solutions of the nonlinear problem can be obtained in a simpler way with reduced calculated terms. Furthermore, El-Zahar [10] introduced an adaptive multistep DTM to obtain solution of singular perturbation initial-value problems. It produces the solution in a rapid convergent series which results in the solution converging in wide time area.

Recently, Hussin et. al [11] proposed and implemented the Multistep Modified Reduced Differential Transform Method (MMRDTM) for solving nonlinear Schrodinger equations (NLSE). The outcome showed the approximate solutions of NLSE with high accuracy were obtained. Hussin et. al [12] also solved Klein-Gordon equations using MMRDTM and results showed that the MMRDTM is a valid and efficient method for finding analytic approximate solution of the Klein-Gordon equations. Besides that, 
Hussin et. al [13] obtain solution of fractional nonlinear Schrodinger equations (FNLSEs) by using MMRDTM.

In this study, we combine the modification made in [9] and the multistep approach in [10] to execute a new technique called Multistep Modified Reduced Differential Transform Method (MMRDTM). The proposed technique has the advantage of yielding an analytical approximation in a fast-convergent sequence with reduced number of computed terms.

\section{The Development of Multistep Modified Reduced Differential Transform Method}

For notation purpose, the original functions will be denoted using lowercase letter such as the letter $\mathrm{u}$ in the function $u(x, t)$, while the transformed functions will be denoted using uppercase letter such as the letter $U$ in the function $U_{k}(x)$. Basically, the differential transformation of the function $u(x, t)=f(x) g(t)$ is given by Keskin and Oturanç [14] as follows,

$$
u(x, t)=\sum_{i=0}^{\infty} F(i) x^{i} \sum_{j=0}^{\infty} G(j) t^{j}=\sum_{k=0}^{\infty} U_{k}(x) t^{k}
$$

where $U_{k}(x)$ is known as the function of $u(x, t)$. Some fundamental properties of RDTM are given as follows:

Definition 1: For an analytically and continuously differential function $u(x, t)$ with respect to time $t$ and space variable $x$, the differential transformation of $u(x, t)$ is defined by

$$
U_{k}(x)=\frac{1}{k !}\left[\frac{\partial^{k}}{\partial t^{k}} u(x, t)\right]_{t=0}
$$

where $U_{k}(x)$ is the transformed function.

Definition 2. The inverse transform of $U_{k}(x)$ is given by

$$
u(x, t)=\sum_{k=0}^{\infty} U_{k}(x) t^{k} .
$$

Then by combining (1) and (2), we can write the equation as following,

$$
u(x, t)=\sum_{k=0}^{\infty} \frac{1}{k !}\left[\frac{\partial^{k}}{\partial t^{k}} u(x, t)\right]_{t=0} t^{k} .
$$

In order to represent the core properties of the RDTM, consider the following nonlinear PDE,

$$
D u(x, t)+P u(x, t)+Q u(x, t)=h(x, t),
$$

where $u(x, 0)=f(x)$ is the initial condition. Here, $D=$ $\frac{\partial}{\partial t}$ and $P$ is the remaining part of linear operator. The nonlinear and inhomogeneous terms are represented as $N u(x, t)$ and $h(x, t)$ respectively.

Based on the MMRDTM, the iteration formula can be formed as follows:

$$
(k+1) U_{k+1}(x)=H_{k}(x)-P U_{k}(x)-N U_{k}(x) .
$$

The

functions $D u(x, t), P u(x, t), N u(x, t)$ and $h(x, t)$ are transformed and then represented as $U_{k}(x), P U_{k}(x), N U_{k}(x)$ and $H_{k}(x)$ respectively. We have

$$
U_{0}(x)=f(x),
$$

from the initial condition. Referring to Ray [9], the nonlinear term is denoted as

$$
N u(x, t)=\sum_{n=0}^{\infty} A_{n}\left(U_{0}(x), U_{1}(x), \ldots, U_{n}(x)\right) .
$$

By combining (6) into (5) and through iterative calculation, the $U_{k}(x)$ values can be obtained. Furthermore, the set of values $\left\{U_{k}(x)\right\}_{k=0}^{n}$ of the inverse transformation yields the following approximate solution,

$$
u(x, t)=\sum_{k=0}^{K} U_{k}(x) t^{k}, t \in[0, T]
$$

The interval $[0, T]$ is divided into $M$ subintervals $\left[t_{i-1}, t_{i}\right]$ for $i=1,2, \ldots, M$, by equal step size $s=\frac{T}{M}$ and nodes $t_{i}=i h$ are used. The following steps are used to calculate MMRDTM. Firstly, the modified RDTM is applied to the initial value problem of interval $\left[0, t_{1}\right]$.

By using the initial conditions

$$
u(x, 0)=f_{0}(x), u_{1}(x, 0)=f_{1}(x),
$$

the approximate result

$$
u_{1}(x, t)=\sum_{k=0}^{K} U_{k, 1}(x) t^{k}, t \in\left[0, t_{1}\right]
$$

is obtained.

At each subinterval $\left[t_{i-1}, t_{i}\right]$, the initial conditions

$$
\begin{aligned}
u_{i}\left(x, t_{i-1}\right) & =u_{i-1}\left(x, t_{i-1}\right), \\
(\partial / \partial t) u_{i}\left(x, t_{i-1}\right) & =(\partial / \partial t) u_{i-1}\left(x, t_{i-1}\right)
\end{aligned}
$$

are used for $i \geq 2$ and the multistep RDTM is implemented to the initial value problem on $\left[t_{i-1}, t_{i}\right]$, where $t_{0}$ is replaced with $t_{i-1}$. For $i=1,2, \ldots, M$, the process is continued and carried out repeatedly to produce a sequence approximate solutions $u_{i}(x, t)$ such as

$$
u_{i}(x, t)=\sum_{k=0}^{K} U_{k, i}(x)\left(t-t_{i-1}\right)^{k}, t \in\left[t_{i-1}, t_{i}\right] .
$$

Finally, the MMRDTM proposes the following solutions:

$$
u(x, t)=\left\{\begin{array}{c}
u_{1}(x, t), \text { for } t \in\left[0, t_{1}\right] \\
u_{2}(x, t), \text { for } t \in\left[t_{1}, t_{2}\right] \\
\vdots \\
u_{M}(x, t), \text { for } t \in\left[t_{M-1}, t_{M}\right] .
\end{array}\right.
$$

With better computing performance, the new algorithm MMRDTM is straightforward for all values of $h$. It can be effortlessly observed that if the step size $h=T$, then the MMRDTM reduces to the modified RDTM. 


\section{Application of MMRDTM for Solving Nonlinear Korteweg-de Vries Equation}

The generalized Korteweg-de Vries (KdV) equation was introduced as follows [15]:

$$
u_{t}+(p+1)(p+2) u^{p} u_{x}+u_{x x x}=g(x, t)
$$

where $g(x, t)$ is a given function with $p=$ $1,2, \ldots$ and $u, u_{x}, u_{x x x} \rightarrow 0$ as $|x| \rightarrow \infty$. For $p=0,1,2$, (7) becomes into the linearized $\mathrm{KdV}$, nonlinear $\mathrm{KdV}$, and modified KdV equation, respectively [16,17].

Applying MMRDTM to (7) and using essential properties of MMRDTM, we can obtain,

$$
\begin{array}{r}
U_{k+1, i}(x)=\left(\frac{1}{(k+1)}\right)(-(p+1)(p+ \\
\text { 2) } \left.\sum_{k=0}^{n} A_{k, i} \frac{\partial}{\partial x}\left(U_{k, i}(x)\right)-\frac{\partial^{3}}{\partial x^{3}} U_{k}(x)+g(x, t)\right) .
\end{array}
$$

From initial condition, we compose

$$
U_{0}(x)=f(x) .
$$

Now, the nonlinear term can be written as follows:

$$
N u(x, t)=\sum_{n=0}^{\infty} A_{n}\left(U_{0}(x), U_{1}(x), \ldots, U_{n}(x)\right) .
$$

Substituting (9) into (8) and by direct iterative computation, we obtain the following $U_{k}(x)$ values. Then, the $n$-terms approximation solution is obtained from the inverse transformation of the set of values $\left\{U_{k}(x)\right\}_{k=0}^{n}$ given as follows:

$$
u(x, t)=\sum_{k=0}^{K} U_{k}(x) t^{k}, t \in[0, T] .
$$

The interval $[0, T]$ is divided into $M$ subintervals $\left[t_{i-1}, t_{i}\right]$ for $i=1,2, \ldots, M$, by equal step size $s=\frac{T}{M}$ and nodes $t_{i}=i h$ are used. The following steps are used to calculate MMRDTM. Firstly, the modified RDTM is applied to the initial value problem of interval $\left[0, t_{1}\right]$.

By using the initial conditions

$$
u(x, 0)=f_{0}(x), u_{1}(x, 0)=f_{1}(x),
$$

the approximate result

$$
u_{1}(x, t)=\sum_{k=0}^{K} U_{k, 1}(x) t^{k}, t \in\left[0, t_{1}\right]
$$

is obtained.

At each subinterval $\left[t_{i-1}, t_{i}\right]$, the initial conditions

$$
\begin{gathered}
u_{i}\left(x, t_{i-1}\right)=u_{i-1}\left(x, t_{i-1}\right), \\
(\partial / \partial t) u_{i}\left(x, t_{i-1}\right)=(\partial / \partial t) u_{i-1}\left(x, t_{i-1}\right)
\end{gathered}
$$

are used for $i \geq 2$ and the multistep RDTM is implemented to the initial value problem on $\left[t_{i-1}, t_{i}\right]$, where $t_{0}$ is replaced with $t_{i-1}$. For $i=1,2, \ldots, M$, the process is continued and carried out repeatedly to produce a sequence approximate solutions $u_{i}(x, t)$ such as

$$
u_{i}(x, t)=\sum_{k=0}^{K} U_{k, i}(x)\left(t-t_{i-1}\right)^{k}, t \in\left[t_{i-1}, t_{i}\right] .
$$

The new algorithm MMRDTM is straightforward for computational performance for all values of $h$. It can be effortlessly observed that if the step size $h=T$, then the MMRDTM reduces to the modified RDTM.

\section{Numerical Results and Discussion}

To represent the effectiveness of the proposed method, we consider three test examples in this section. For accuracy evaluation purpose, we compare the obtained solutions with the exact solutions for each example.

Example 1: Consider the KdV equation which takes the form $[18,19]$ :

$$
u_{t}+6 u u_{x}+u_{x x x}=0, x \in \mathbb{R}
$$

subject to the initial condition

$$
u(x, 0)=\frac{1}{2} \operatorname{sech}^{2}\left(\frac{x}{2}\right)
$$

The exact solution of this equation is $\frac{1}{2} \operatorname{sech}^{2}\left(\frac{x-t}{2}\right)$. Using basic properties of MMRDTM then applying MMRDTM to (10), we can obtain

$$
\begin{aligned}
U_{k+1, i}(x) & =\left(\frac{1}{(k+1)}\right)\left(-6 \sum_{r=0}^{k} U_{k-r, i}(x) \frac{\partial}{\partial x} U_{r, i}(x\right. \\
) & \left.-\frac{\partial^{3}}{\partial x^{3}}\left(U_{k, i}(x)\right)\right)
\end{aligned}
$$

From the initial condition, we express

$$
U_{0}(x)=\frac{1}{2} \operatorname{sech}^{2}\left(\frac{x}{2}\right) .
$$

The $U_{k}(x)$ values were obtained by substituting (12) into (11) by straightforward iterative calculation.

The interval $[0,1]$ is divided into 10 subintervals $\left[t_{i-1}, t_{i}\right]$ for $i=1,2, \ldots, 10$, by equal step size $h=0.1$ and nodes $t_{i}=i h$ are used. Firstly, the modified RDTM is applied to the initial value problem of interval $\left[0, t_{1}\right]$.

By using the initial conditions

$$
u(x, 0)=f_{0}(x), u_{1}(x, 0)=f_{1}(x),
$$

the approximate result

$$
u_{1}(x, t)=\sum_{k=0}^{K} U_{k, 1}(x) t^{k}, t \in\left[0, t_{1}\right]
$$

is obtained.

At each subinterval $\left[t_{i-1}, t_{i}\right]$, the initial conditions

$$
u_{i}\left(x, t_{i-1}\right)=u_{i-1}\left(x, t_{i-1}\right) \text {, }
$$




$$
(\partial / \partial t) u_{i}\left(x, t_{i-1}\right)=(\partial / \partial t) u_{i-1}\left(x, t_{i-1}\right)
$$

are used for $i \geq 2$ and the multistep RDTM is implemented to the initial value problem on $\left[t_{i-1}, t_{i}\right]$, where $t_{0}$ is replaced with $t_{i-1}$. For $i=1,2, \ldots, 10$, the process is continued and carried out repeatedly to produce a sequence approximate solutions $u_{i}(x, t)$ such as

$$
u_{i}(x, t)=\sum_{k=0}^{K} U_{k, i}(x)\left(t-t_{i-1}\right)^{k}, t \in\left[t_{i-1}, t_{i}\right] .
$$

The exact solution, approximate solutions yielded by the MMRDTM for $t \in[-2,2], x \in[0,1]$ and the approximate solution given by the RDTM for $t \in[-2,2], x \in[0,1]$ for the Example 1 are graphically represented in the Fig. 1A, Fig. $1 \mathrm{~B}$ and Fig.1C respectively. Observe that, the multistep modified approximate solutions for this type of nonlinear $\mathrm{KdV}$ equations obtain the exact solutions while the approximate solution of RDTM is convergent to the exact solution as in [19-21]. The performance error analyses obtained by MMRDTM and RDTM are summarized in Table 1.

Table 1. Comparison error results of MMRDTM and RDTM approximate solutions for Example 1

\begin{tabular}{|c|c|c|c|}
\hline $\mathbf{T}$ & Exact Solution & MMRDTM & $\begin{array}{c}\text { Absolute Error } \\
\text { (RDTM) }\end{array}$ \\
\hline 0.1 & 0.500000000 & 0.500000000 & 0.00000000 \\
\hline 0.2 & 0.5000000000 & 0.500000000 & $6.500000010^{-9}$ \\
\hline 0.3 & 0.5000000000 & 0.500000000 & $1.542000010^{-7}$ \\
\hline 0.4 & 0.5000000000 & 0.500000000 & $1.385500010^{-6}$ \\
\hline 0.5 & 0.5000000000 & 0.500000000 & $7.185200010^{-6}$ \\
\hline 0.6 & 0.5000000000 & 0.500000000 & $2.595300010^{-5}$ \\
\hline 0.7 & 0.5000000000 & 0.500000000 & $7.198500010^{-5}$ \\
\hline 0.8 & 0.5000000000 & 0.500000000 & $1.618951010^{-4}$ \\
\hline 0.9 & 0.5000000000 & 0.500000000 & $3.038343010^{-4}$ \\
\hline 1.0 & 0.5000000000 & 0.500000000 & $4.793778010^{-4}$ \\
\hline
\end{tabular}

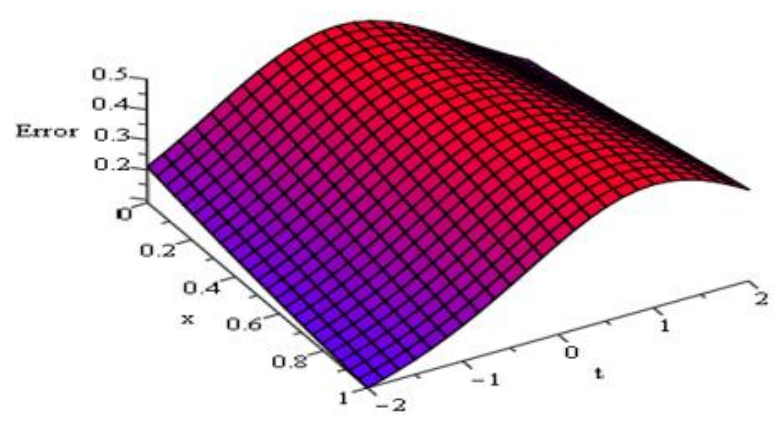

Figure 1A. Exact solution

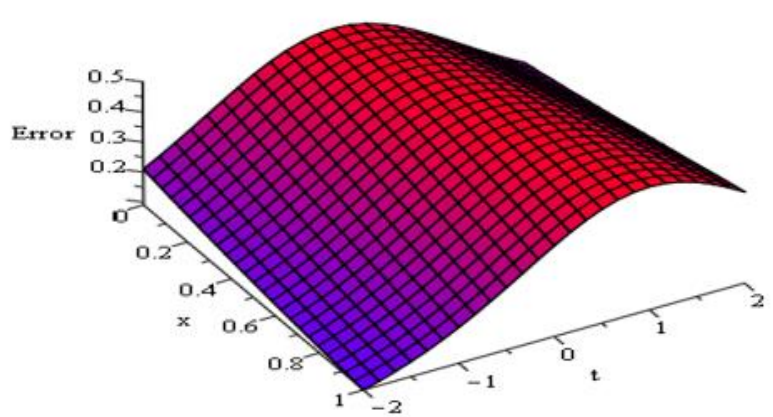

Figure 1B. MMRDTM

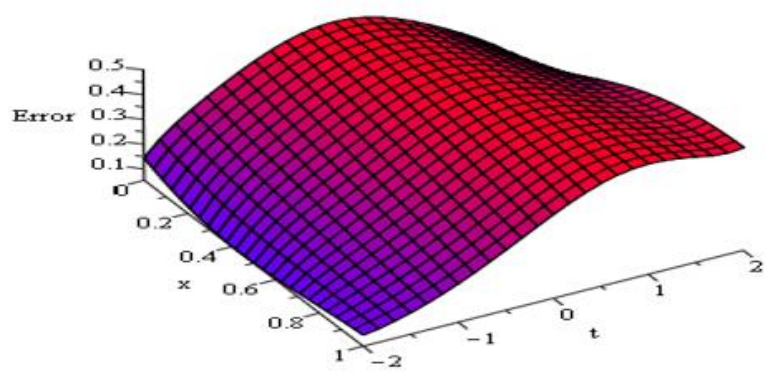

Figure 1C. RDTM

Example 2: Consider the KdV equation which has twosoliton solution [9]

$$
u_{t}+6 u u_{x}+u_{x x x}=0, x \in \mathbb{R}
$$

with respect to the initial condition

$$
u(x, 0)=6 \operatorname{sech}^{2} x .
$$

The exact solution of this equation is

$$
\frac{24\left(4 \cosh (x-4 t)^{2}+\sinh (2 x-32 t)^{2}\right)}{(\cosh (3 x-36 t)+3 \cosh (x-28 t))^{2}} \text {. }
$$

Using basic properties of MMRDTM then applying MMRDTM (13), we can obtain

$$
\begin{gathered}
U_{k+1, i}(x)=\left(\frac{1}{(k+1)}\right)\left(-6 \sum_{r=0}^{k} U_{k-r, i}(x) \frac{\partial}{\partial x} U_{r, i}(x\right. \\
)-\frac{\partial^{3}}{\partial x^{3}}\left(U_{k, i}(x)\right)\right)
\end{gathered}
$$

From initial condition, write

$$
U_{0}(x)=6 \operatorname{sech}^{2} x .
$$

The interval $[0,1]$ is divided into 10 subintervals $\left[t_{i-1}, t_{i}\right]$ for $i=1,2, \ldots, 10$, by equal step size $h=0.1$ and nodes $t_{i}=i h$ are used. Firstly, the modified RDTM is applied to the initial value problem of interval $\left[0, t_{1}\right]$.

By using the initial conditions

$$
u(x, 0)=f_{0}(x), u_{1}(x, 0)=f_{1}(x),
$$

the approximate result 


$$
u_{1}(x, t)=\sum_{k=0}^{K} U_{k, 1}(x) t^{k}, t \in\left[0, t_{1}\right]
$$

is obtained.

At each subinterval $\left[t_{i-1}, t_{i}\right]$, the initial conditions

$$
\begin{aligned}
u_{i}\left(x, t_{i-1}\right) & =u_{i-1}\left(x, t_{i-1}\right), \\
(\partial / \partial t) u_{i}\left(x, t_{i-1}\right) & =(\partial / \partial t) u_{i-1}\left(x, t_{i-1}\right)
\end{aligned}
$$

are used for $i \geq 2$ and the multistep RDTM is implemented to the initial value problem on $\left[t_{i-1}, t_{i}\right]$, where $t_{0}$ is replaced with $t_{i-1}$. For $i=1,2, \ldots, 10$, the process is continued and carried out repeatedly to produce a sequence approximate solutions $u_{i}(x, t)$ such as

$$
u_{i}(x, t)=\sum_{k=0}^{K} U_{k, i}(x)\left(t-t_{i-1}\right)^{k}, t \in\left[t_{i-1}, t_{i}\right] .
$$

Fig. 2A shows the exact solution, Fig. 2B shows graph of approximate solution of MMRDTM for $t \in[-2,2]$ and $x$ $\in[-6,6]$ while Fig. 2C shows graph of approximate solution RDTM for $t \in[-2,2]$ and $x \in[-6,6]$. Clearly, the multistep modified approximate solutions for this type of nonlinear $\mathrm{KdV}$ equations converge to the exact solutions as in [9].

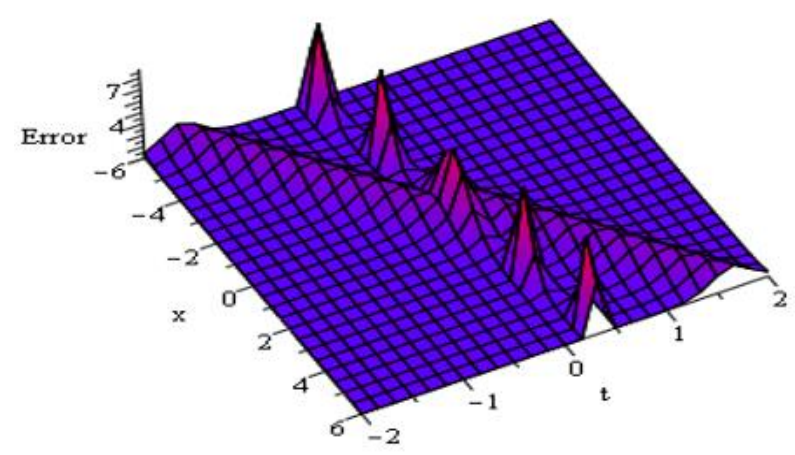

Figure 2A. Exact solution

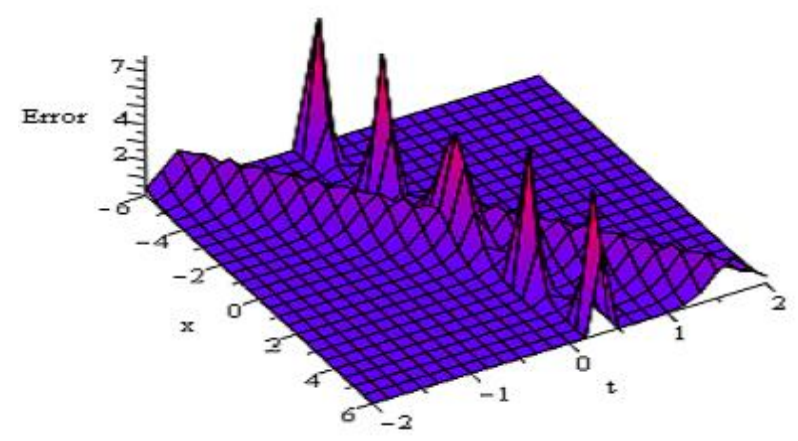

Figure 2B. MMRDTM

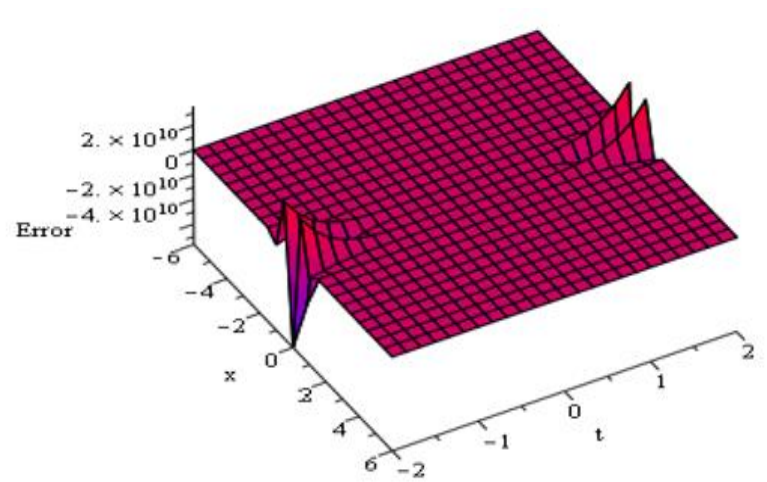

Figure 2C. RDTM

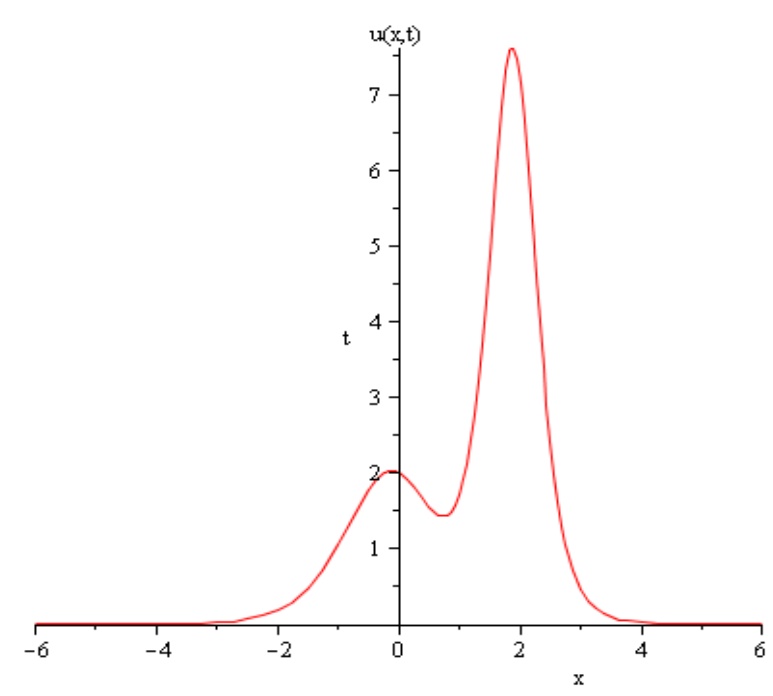

Figure 2D. 2-Dimensional graph for Exact Solution

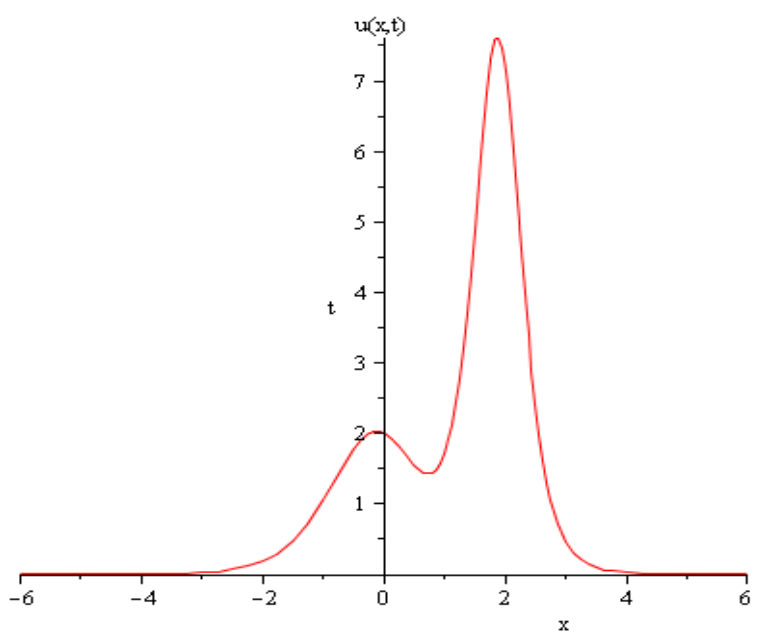

Figure 2E. 2-Dimensional graph for MMRDTM 


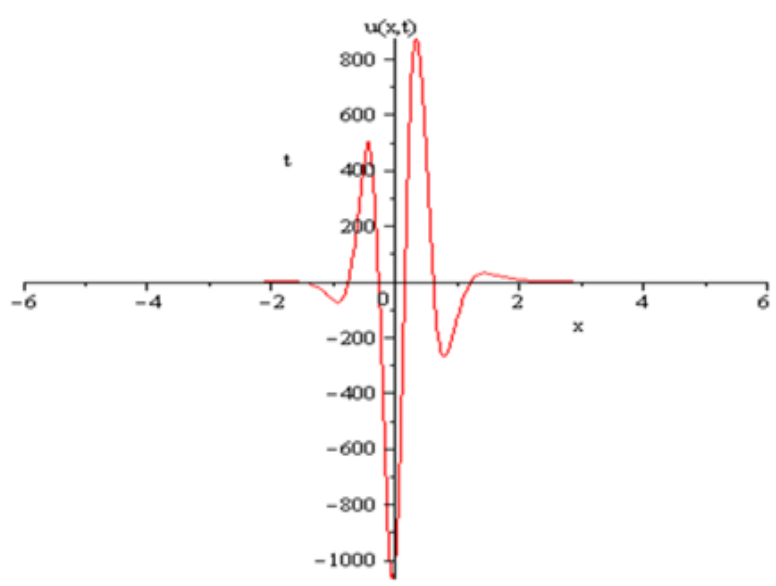

Figure 2F. 2-Dimensional graph for RDTM

Example 3 Consider the nonlinear $\mathrm{KdV}$ equation of the form [19]

$$
u_{t}+6 u^{2} u_{x}+u_{x x x}=0, x \in \mathbb{R}
$$

subject to the initial condition

$$
u(x, 0)=\operatorname{sech}(x)
$$

where the exact solution of this equation is sech $(x-t)$.

Using basic properties of MMRDTM and then applying MMRDTM to (16), we obtain

$$
\begin{gathered}
U_{k+1, i}(x)=\left(\frac{1}{(k+1)}\right)(-6 \\
\sum_{s=0}^{k} \sum_{l=0}^{s} U_{s-l, i}(x) U_{k-s, i}(x) \frac{\partial}{\partial x}\left(U_{l, i}(x)\right)- \\
\left.\frac{\partial^{3}}{\partial x^{3}}\left(U_{k, i}(x)\right)\right) .
\end{gathered}
$$

From the initial condition, write

$$
U_{0}(x)=\operatorname{sech}(x) .
$$

The interval $[0,1]$ is divided into 10 subintervals $\left[t_{i-1}, t_{i}\right]$ for $i=1,2, \ldots, 10$, by equal step size $h=0.1$ and nodes $t_{i}=i h$ are used. Firstly, the modified RDTM is applied to the initial value problem of interval $\left[0, t_{1}\right]$.

By using the initial conditions

$$
u(x, 0)=f_{0}(x), u_{1}(x, 0)=f_{1}(x),
$$

the approximate result

$$
u_{1}(x, t)=\sum_{k=0}^{K} U_{k, 1}(x) t^{k}, t \in\left[0, t_{1}\right]
$$

is obtained.

At each subinterval $\left[t_{i-1}, t_{i}\right]$, the initial conditions

$$
\begin{aligned}
u_{i}\left(x, t_{i-1}\right) & =u_{i-1}\left(x, t_{i-1}\right), \\
(\partial / \partial t) u_{i}\left(x, t_{i-1}\right) & =(\partial / \partial t) u_{i-1}\left(x, t_{i-1}\right)
\end{aligned}
$$

are used for $i \geq 2$ and the multistep RDTM is implemented to the initial value problem on $\left[t_{i-1}, t_{i}\right]$, where $t_{0}$ is replaced with $t_{i-1}$. For $i=1,2, \ldots, 10$, the process is continued and carried out repeatedly to produce a sequence approximate solutions $u_{i}(x, t)$ such as

$$
u_{i}(x, t)=\sum_{k=0}^{K} U_{k, i}(x)\left(t-t_{i-1}\right)^{k}, t \in\left[t_{i-1}, t_{i}\right] .
$$

Fig. 3A shows the exact solution, Fig. 3B shows graph of approximate solution of MMRDTM for $t \in[-1,1]$ and $x$ $\in[0,1]$ while Fig. 3C shows graph of approximate solution RDTM for $t \in[-1,1]$ and $x \in[0,1]$. It can be observed that the multistep modified approximate solutions for this type of nonlinear $\mathrm{KdV}$ equations obtain the exact solutions while the approximate solution of RDTM is convergent to the exact solution as in [19-21]. The performance error analyses obtained by MMRDTM and RDTM are summarized in Table 2.

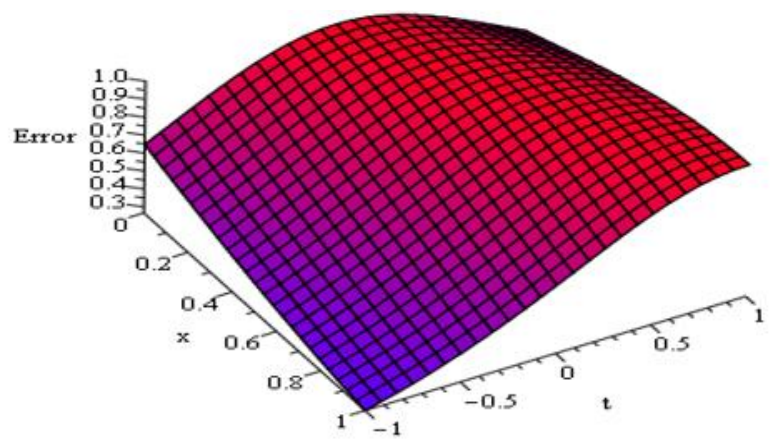

Figure 3A. Exact solution

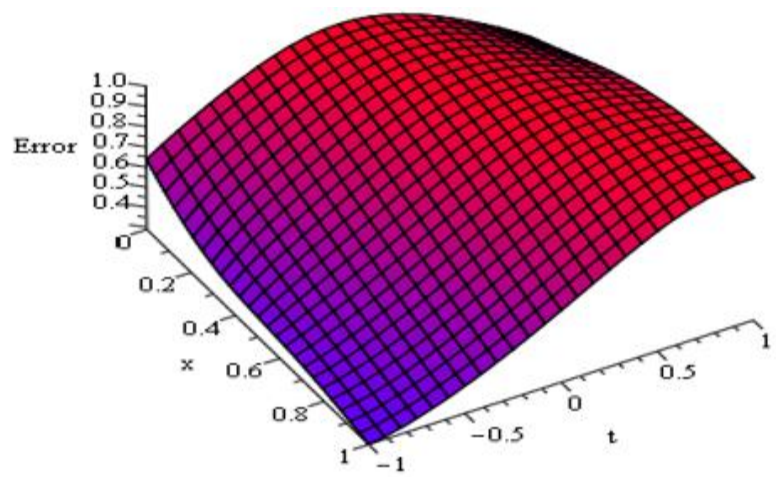

Figure 3B. MMRDTM

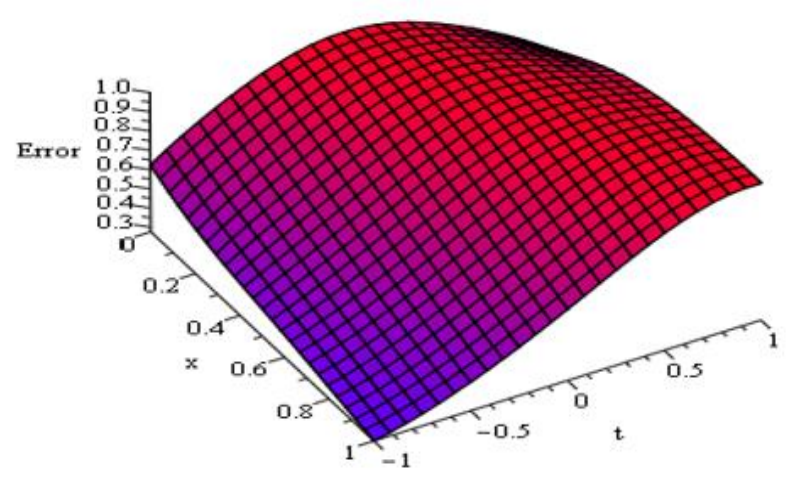

Figure 3C. RDTM 
Table 2. Comparison error results of MMRDTM and RDTM approximate solutions for Example 3.

\begin{tabular}{|c|c|c|c|}
\hline T & Exact Solution & MMRDTM & $\begin{array}{c}\text { Absolute Error } \\
\text { (RDTM) }\end{array}$ \\
\hline 0.1 & 1.0000000000 & 1.00000000 & $3.0000000010^{-9}$ \\
\hline 0.2 & 1.0000000000 & 1.00000000 & $5.0600000010^{-7}$ \\
\hline 0.3 & 1.0000000000 & 1.00000000 & $1.0087000010^{-5}$ \\
\hline 0.4 & 1.0000000000 & 1.00000000 & $6.9869000010^{-5}$ \\
\hline 0.5 & 1.0000000000 & 1.00000000 & $2.5044200010^{-4}$ \\
\hline 0.6 & 1.0000000000 & 1.00000000 & $5.1965000010^{-4}$ \\
\hline 0.7 & 1.0000000000 & 1.00000000 & $4.8411500010^{-4}$ \\
\hline 0.8 & 1.0000000000 & 1.00000000 & $7.8479640010^{-4}$ \\
\hline 0.9 & 1.0000000000 & 1.00000000 & $4.5886228010^{-3}$ \\
\hline 1.0 & 1.0000000000 & 1.00000000 & $1.2074817410^{-2}$ \\
\hline
\end{tabular}

\section{Conclusions}

We proposed an approximate analytical method called the MMRDTM in this paper. In the proposed method, the modification involves the substitution of nonlinear term by its Adomian polynomials and the use of a multistep approach. The significance of the modification is that for any analytical nonlinearity, the nonlinear $\mathrm{KdV}$ term can be easily solved with less computational work. This is due to the Adomian polynomials' properties and available algorithms. To illustrate the effectiveness of the proposed method, we executed the method for obtaining the solution of the one-dimensional nonlinear equations of $\mathrm{KdV}$. Moreover, we also implemented the multistep RDTM which offers accurate approximate solutions over longer time frames. As a result, the approximate solutions of nonlinear $\mathrm{KdV}$ equation obtained with high precision and good agreement to the exact solutions. Moreover, the proposed method also more accurate and easier to solve compared to RDTM. We conclude that, the MMRDTM is valid and it offers good efficiency for obtaining analytic approximate solution for these types of equations. The computations in this paper were solved by utilizing Maple 13.

\section{Acknowledgements}

The authors express their appreciation of supporting this research to the Malaysian Higher Education Ministry and Universiti Malaysia Sabah. We also have Universiti Sains Malaysia financial support.

\section{REFERENCES}

[1] L. Debtnath. Nonlinear Partial Differential Equations for
Scientist and Engineers, Birkhauser, Boston, 1997.

[2] A. F. Jameel, N. R. Anakira, M. M. Rashidi, A. K. Alomari, A. Saaban, M. A. Shakhatreh. Differential Transformation Method for Solving High Order Fuzzy Initial Value Problems, Italian Journal of Pure and Applied Mathematics, Vol.39, 194-208, 2018.

[3] T. R. R. Rao. Numerical Solution of Sine Gordon Equations Through Reduced Differential Transform Method, Global Journal of Pure and Applied Mathematics, Vol.13, No.7, 3879-3888, 2017.

[4] O. Acan, Y. Keskin. Reduced Differential Transform Method for $(2+1)$ Dimensioal Type of the ZakharovKuznetsov ZK (n,n) Equations, AIP Conference Proceedings, Vol.1648, No. 1, 2015.

[5] H. R. Marasi, N. Sharifi, H. Piri. Modified Differential Transform Method for Singular Lane-Emden Equations in Integer and Fractional Order, Journal of Applied and Engineering Mathematics, Vol.5, No.1, 124-131, 2015.

[6] B. Benhammouda, H. V. A. Leal. New Multi - Step Technique with Differential Transform Method for Analytical Solution of Some Nonlinear Variable Delay Differential Equations, SpringerPlus, Vol. 5, No. 1, 1723,2016 .

[7] S. Sahoo, S. S. Ray. Solitary wave solutions for time fractional third order modified KdV equation using two reliable techniques $\left(\mathrm{G}^{\prime} / \mathrm{G}\right)$-expansion method and improved (G'/G)-expansion method, Physica A., Vol. 448, 265-282, 2015.

[8] M. S. Islam, K. Khan, M. A. Akbar. An analytical method for finding exact solutions of modified Korteweg - de Vries equation, Results in Physics, Vol. 5, 131-135, 2015.

[9] S. S. Ray. Numerical Solutions and Solitary Wave Solutions of Fractional KdV Equations using Modified Fractional Reduced Differential Transform Method, Computational Mathematics and Mathematical Physics, Vol. 53, No. 12, 1870-1881, 2013.

[10] E. R. El-Zahar. Applications of Adaptive Multi Step Differential Transform Method to Singular Perturbation Problems Arising in Science and Engineering, Applied Mathematics and Information Sciences, Vol. 9, No.1, 223232, 2015.

[11] C. H. C. Hussin, A. Kilicman, A. Azmi. Analytical Solutions of Nonlinear Schrodinger Equations using Multi-step Modified Reduced Differential Transform Method, International Journal of Advanced Computer Technology, Vol.7, No.11, 2939-2944, 2018.

[12] C. H. C. Hussin, A. I. M. Ismail, A. Kilicman, A. Azmi. Analytical Solutions of Non-Linear Klein-Gordon Equations Using Multistep Modified Reduced Differential Transform Method, Thermal Science, Vol.23, No.1, S317S326, 2019.

[13] C. H. C. Hussin, A. I. M. Ismail, A. Kilicman, A. Azmi. Approximate Analytical Solutions of Fractional Nonlinear Schrodinger Equations using Multistep Modified Reduced Differential Transform Method, Proceedings of the International Conference on Mathematical Sciences and Technology 2018 (MathTech2018), AIP Conference Proceedings, Vol.2184, 060003, 2019. 
[14] Y. Keskin, G. Oturanc. Reduced Differential Transform Method for Partial Differential Equations, International Journal of Nonlinear Sciences and Numerical Simulation, Vol.10, No.6, 741-749, 2009.

[15] P. G. Drazin, R. S. Johnson. Solitons: An Introduction, Cambridge University Press, Cambridge, 1989.

[16] D. Kaya, M. Aassila. An application for a generalized KdV equation by the decomposition method, Physics Letters A, Vol.299, 201-206, 2002.

[17] S. Momani, Z. Odibat, A Alawneh. Variational Iteration Method for Solving the Space- and Time-Fractional KdV Equation, Numerical Methods for Partial Differential Equations, Vol. 24, No.1, 262-271, 2007.

[18] P. Saucez, A. V. Wouwer, W. E. Schiesser. An adaptive method of lines solution of the Korteweg-de Vries equation, Computers and Mathematics with Applications, Vol.35, No.12, 13-25, 1998.

[19] Y. Keskin, G. Oturanc. Reduced Differential Transform Method for Generalized KDV Equations, Mathematical and Computational Applications, Vol.15, No.3, 382-393, 2010.

[20] D. Kaya, M. Aassila. An application for a generalized KdV equation by the decomposition method, Physics Letters A, Vol. 299, 201-206, 2002.

[21] A.T. Abassy, M. A. El-Tawil, H. El. Zoheiry. Solving nonlinear partial differential equations using the modified variational iteration Pade technique, Journal of Computational and Applied Mathematics, Vol. 207, No. 1, 73-91, 2007. 